\title{
Irradiation Induced Localized Amorphization in Mo-Re Alloy Films
}

\author{
Yin $\mathrm{Hu}$, Zhengcao $\mathrm{Li}^{*}$ and Zhengjun Zhang \\ Advanced Materials Laboratory, Department of Materials Science and Engineering, \\ Tsinghua University, Beijing 100084, P.R. China
}

\begin{abstract}
To investigate the irradiation properties of Mo-Re alloy of low Re content (atm\%: 0\% 8.4\%), Mo-Re alloy films were irradiated by $200 \mathrm{keV}$ xenon ions at the dose of $5 \times 10^{15}$ ions $/ \mathrm{cm}^{2}$. Different irradiation effects were observed on the samples of different Re content. While the sample of pure Mo remained the same after irradiation, obvious localized amorphization phenomenon took place in the sample with Re content of $8.4 \%$. So it is possible that Mo-Re alloy with low Re content might break down for the irradiation induced amorphization process. The formation mechanism of amorphous regions was then discussed by irradiation experiments at ion dose from $1 \times 10^{15}$ to $5 \times 10^{15}$ ions $/ \mathrm{cm}^{2}$, by which the amorphization process developing from lattice damages to amorphous clusters, and finally to amorphous regions, was connected with the increase of irradiation dose step by step. [doi:10.2320/matertrans.M2009306]
\end{abstract}

(Received September 7, 2009; Accepted January 22, 2010; Published March 17, 2010)

Keywords: molybdenum-rhenium alloy, ion irradiation, lattice damages, localized amorphization

As one of the most important results for the implantation of heavy ions, the irradiation induced amorphization have been of great interests. Experiments have shown that many crystalline materials undergone the crystalline-to-amorphous transformation under ion beam irradiation. ${ }^{1-5)}$ On the other hand, remarkable changes always take place in material property during the amorphization process. ${ }^{6-10)}$ For example, McHargue and co-workers reported that the hardness and elastic modulus values of amorphous $\mathrm{SiC}$ are $40 \%$ and $70 \%$, respectively, of the unimplanted single crystalline values, ${ }^{6}$ Tanab et al. reported the irradiated amorphization effects in graphite such as the increase of hydrogen retention and the loss of thermal conductivity, ${ }^{7)}$ Hioki et al. reported that the surface amorphization reduced the hardness of implanted sapphire, and considerably increased the surface plasticity, ${ }^{8)}$ and Iwaki et al. found that ion beam bombardment to polymers also increased the electrical conductivity of polyimide films for the amorphization effects. ${ }^{9)}$ Therefore irradiation induced amorphization is considered as one of the main factors in ion beam fabrication and irradiation modification.

The positive effects of Rhenium additions on the mechanical behaviors of Molybdenum alloys have been known for decades. Rhenium enhances the Mo-Re alloy both in hightemperature strength and low temperature ductility. ${ }^{10,11)}$ For their outstanding applied capability, various Mo-Re alloys (Re content from $5 \%$ to $31.8 \%$ ) are considered as attractive candidates as fuel cladding and core structural materials in nuclear reactor applications. On the other hand, the ion irradiation effects are key factors in nuclear applications which could induce to irradiation damage and amorphization, so the irradiation properties of Mo-Re alloys should be considered and checked before such applications. ${ }^{12}$ )

As reported, the irradiation effects of intermetallic compounds are sometimes quite different from pure metal. ${ }^{4)}$ In Mo-Re system, radiation-enhanced segregation and precipitation which could ultimately lead to radiation-induced embrittlement have already been observed in Mo-Re alloys with rhenium contents of $26.4 \sim 31.8 \% .^{13,14)}$ Otherwise, the

*Corresponding author, E-mail: zcli@tsinghua.edu.cn irradiation effect of Mo-Re alloys with low rhenium contents $(<10 \%)$ are still unclear. $\left.{ }^{15}\right)$

In this Letter, the irradiation effects of Mo-Re alloy films were studied by $200 \mathrm{keV} \mathrm{Xe}{ }^{+}$implantation. It is found that localized amorphization phenomena were observed in Mo8.4Re film while pure Mo film kept unchanged. Therefore the increase of $\mathrm{Re}$ content in Mo-Re alloy may induce to the trend of amorphization in irradiation treatment. To investigate the principle of amorphization process in Mo-8.4Re films, a series of implantation experiments at different ion doses were carried out, and a route of amorphization process in Mo-8.4Re alloy films was set up by contacting different amorphization phenomena to the corresponding ion dose.

At first, Mo-Re alloy films were obtained by magnetron sputtering. While targets of pure Mo and Re were put together and sputtered at the same time, uniform Mo-Re alloy films were deposited onto the freshly cleaved monocrystal $\mathrm{NaCl}$ substrates. The former operations were taken in an ultra high vacuum system with a background vacuum level of $1.3 \times 10^{-6} \mathrm{~Pa}$. By selecting experimental parameters, the alloy films were designed with a thickness of $40 \mathrm{~nm}$, which was to match the projected range plus the projected range straggling of the $200 \mathrm{keV}$ xenon ions used in irradiation experiments. Different compositions of the alloy samples were obtained by adjusting the sputtered area of Mo and Re targets.

X-ray fluorescence spectroscopy (XRF) analysis was employed to confirm the compositions of the as-deposited samples. Irradiation experiments were then carried out at room temperature by $200 \mathrm{keV} \mathrm{Xe}^{+}$in an implanter with a vacuum level of $1.3 \times 10^{-4} \mathrm{~Pa}$. The ion current density was confined to $3 \mathrm{~mA} / \mathrm{cm}^{2}$, in order to minimize the beam heating effect. All the studied Mo-Re films were removed from $\mathrm{NaCl}$ substrates in de-ionized water and put on $\mathrm{Cu}$ grids. Then the Mo-Re films were sent for transmission electron microscopy (TEM) examination and selected area diffraction (SAD) analysis to identify the resultant morphology and structure.

In Fig. 1, we present the experimental results to discuss the irradiation effects on Mo-Re alloy film. After magnetron sputtering, XRF was used to determine the Re content in the films. Two typical TEM images of the alloy films are shown 


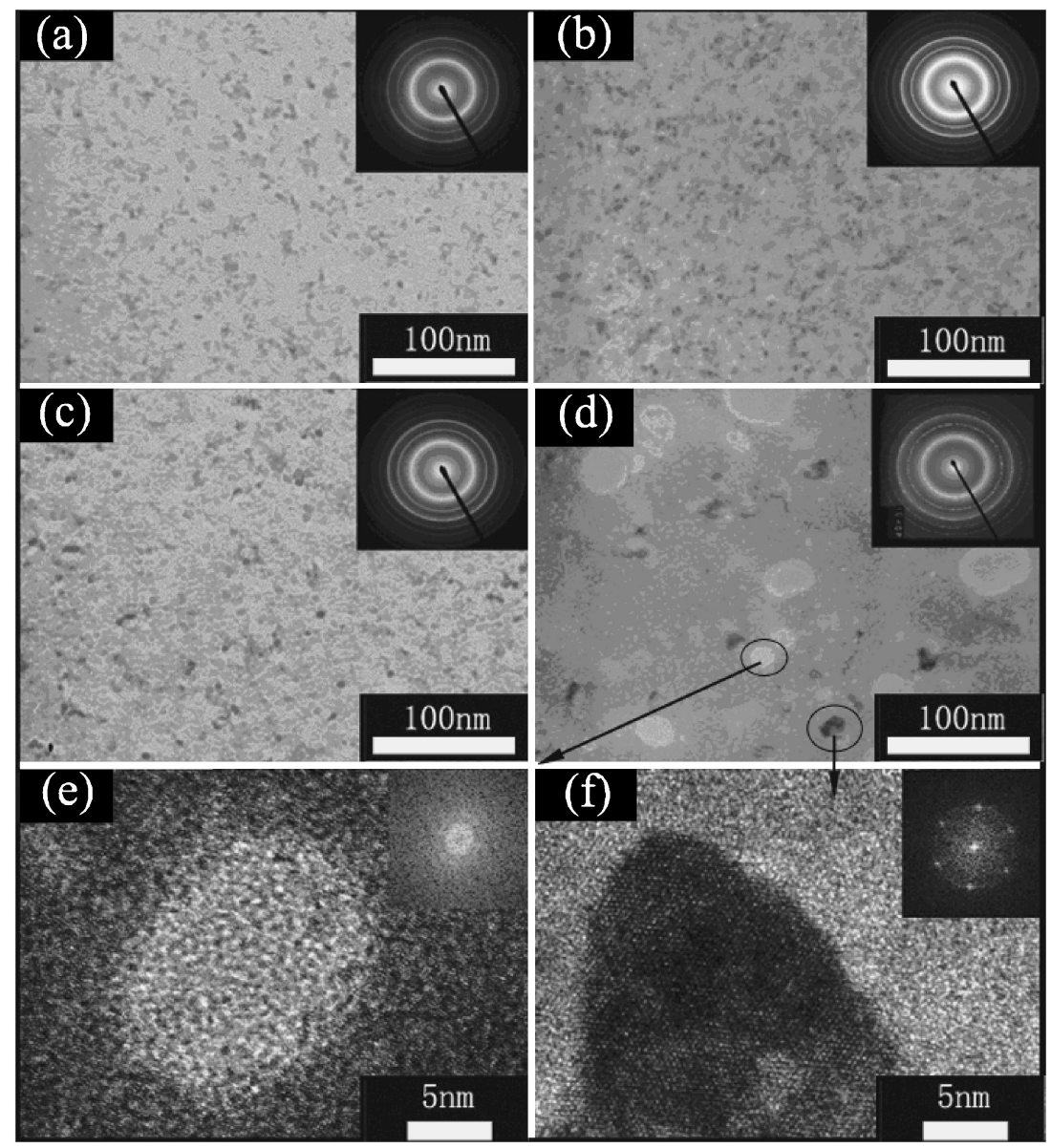

Fig. 1 (a) and (b) show the TEM images of pure Mo and Mo-8.4Re film fabricated by magnetron sputtering; after irradiation by $200 \mathrm{keV}$ $\mathrm{Xe}^{+}$at the dose of $5 \times 10^{15}$ ions $/ \mathrm{cm}^{2}$, the films changed to (c) pure Mo and (d) Mo-8.4Re; in order to show the details in Fig. 1(d), two HRTEM images of (e) and (f) were taken on the amorphous region and crystal gain, while the results of Fourier transform were inserted respectively.

in Fig. 1(a) and 1(b), while the Re contents were respectively measured as $0 \%$ and $8.4 \%$ by XRF. We could observe that the morphology of the films keep the same with different $\mathrm{Re}$ contents, both films are made up of nano-scale crystal grains. According to the SAD patterns, both films fit well with the Mo (bcc) structure. So the film of Mo-8.4Re should be considered as the solid dissolution of Re element in Mo (bcc).

After irradiated by $200 \mathrm{keV} \mathrm{Xe}^{+}$at the dose of $5 \times$ $10^{15}$ ions $/ \mathrm{cm}^{2}$, the morphology and structure of the alloy films are shown in Fig. 1(c) and 1(d). In Fig. 1(c), we could see that no changes took place after irradiation in the films of pure Mo. According to the SAD patterns, the ion implantation did not change the structure of the film either. Interestingly, when increasing the content of Re, different irradiation effects were observed. Figure 1(d) shows the sample of Mo-8.4Re after irradiation. Although the SAD patterns show that the overall structure of the alloy film kept the same, obvious changes including white and black regions could be observed in the film.

To investigate the changes taken place in irradiated Mo8.4Re film, High resolution TEM (HRTEM) images of the white and black regions in Mo-8.4Re alloy film were taken and Fourier transformation of selected area were carried out on those images. One of the white regions is shown in Fig. 1(e), the morphology and the calculated SAD patterns (by Fourier transformation) indicate that it is an amorphous region with the diameter of $\sim 10 \mathrm{~nm}$. On the other hand, Fig. 1(f) proves that the black regions are crystal grains of Mo (bcc) structures, with the diameter of $\sim 20 \mathrm{~nm}$.

The Re contents of the amorphous regions and the crystal grains were then detected by EDS analysis and the composition of the films came out to be Mo5.8Re and Mo10.1Re. So we could say that the amorphous regions are lack of $\mathrm{Re}$ and the crystal grains are rich of $\mathrm{Re}$ according to the average Re content of $8.4 \%$.

To summarize, the irradiation effects of Mo-Re alloy films were expressed as follows:

$$
\begin{aligned}
& \text { Pure Mo } \stackrel{5 \times 10^{15} \mathrm{Xe}^{+} / \mathrm{cm}^{2}}{\longrightarrow} \text { No changes } \\
& \text { Mo8.4Re } \stackrel{5 \times 10^{15} \mathrm{Xe}^{+} / \mathrm{cm}^{2}}{\longrightarrow}\left\{\begin{array}{l}
\text { Crystal grains } \\
(\text { Mo10.1Re }) \\
\\
\text { Amorphous regions } \\
(\text { Mo5.8Re })
\end{array}\right.
\end{aligned}
$$

After the irradiation of $200 \mathrm{keV} \mathrm{Xe}{ }^{+}$at the dose of $5 \times 10^{15}$ ions $/ \mathrm{cm}^{2}$, alloy films with different composition showed different irradiation properties. While the film of pure Mo kept unchanged, the irradiation induced growth of amorphous regions and crystal grains could be clearly observed in the Mo-8.4Re film. So the addition of $\operatorname{Re}$ to 

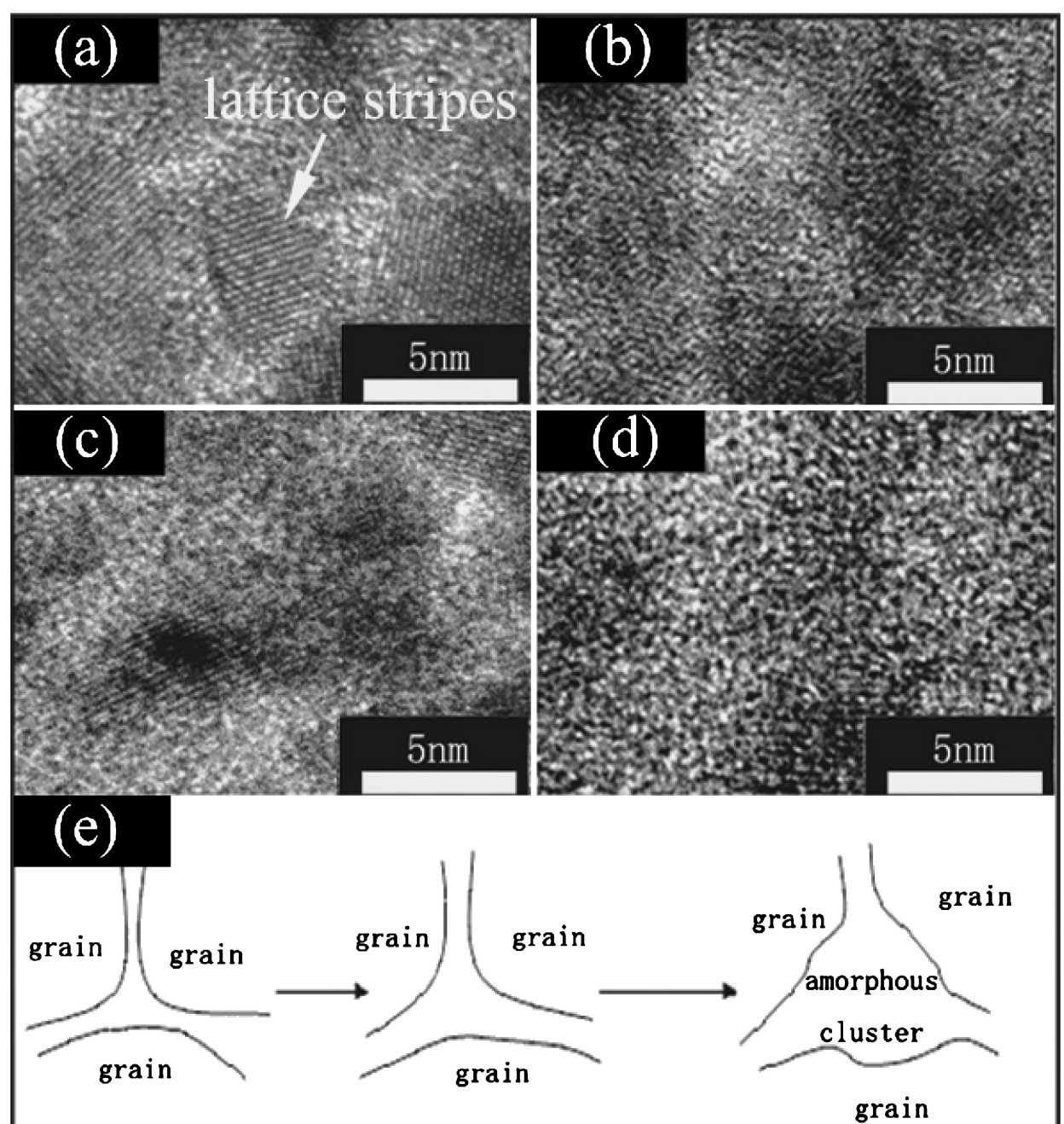

Fig. 2 HRTEM images of Mo-8.4Re film irradiated at different doses: (a) unirradiated; (b) $1 \times 10^{15}$ ions $/ \mathrm{cm}^{2},(\mathrm{c}) 2 \times 10^{15}$ ions $/ \mathrm{cm}^{2}$, and (d) $3 \times 10^{15}$ ions $/ \mathrm{cm}^{2}$; to explain the forming process of amorphous clusters from the collapse of the crastal grains, a sketch is shown in $(\mathrm{e})$.

$8.4 \%$ (atm\%) changed the irradiation properties of the Mo-Re alloy films, and the Re content might be even harmful for the irradiation induced amorphization.

As reported, the growth of grains and precipitates in MoRe alloys could be induced by the irradiation of neutron or heavy ions. ${ }^{13,14)}$ This phenomenon could be explained by the energy deposition process which is induced by the implantation of high energy particles: while particles slow down in the film, they pass energy to the film and induce the crystallization or precipitation process.

On the other hand, irradiation induced amorphization in Mo-Re system has not yet been widely noticed. In fact, simulations on Mo-Re alloy system indicated that the transformation should be non-spontaneous in thermodynamics. However, the implantation of $\mathrm{Xe}^{+}$could be considered as a non-equilibrium process, in which some non-spontaneous reactions in thermodynamics may be carried out. ${ }^{4)}$ In our case, the Mo-Re alloy film of $40 \mathrm{~nm}$ was irradiated by $200 \mathrm{keV} \mathrm{Xe} \mathrm{Xe}^{+}$to a dose of $5 \times 10^{15} \mathrm{ions} / \mathrm{cm}^{2}$. The total irradiation damage is calculated to be $89.16 \mathrm{dpa}$ (displacements per atom), thus it is reasonable that the high irradiation damage level has induced the localized amorphization in the Mo-Re system.
Otherwise, the addition of Re could weaken the steady structure of pure Mo matrix, and the Mo-Re film with a Re content of $8.4 \%$ shows radiative sensitivity in the irradiation process. Since the Re atoms in Mo matrix are heavier than the Mo atoms, they are much harder to transfer into the amorphous area in the grain boundaries. In the amorphization process, $\mathrm{Re}$ atoms prefer to remain in the matrix and the Mo atoms are easier to migrate to the amorphous area. As a result, the Re concentration in amorphous regions is lower than that in crystal grain precipitated in the irradiation process.

While being applied, the irradiation effects of Mo-Re alloys must be cared. Once the irradiation effects become remarkable, corresponding treatments should be adopted in time. So it is necessary to investigate the irradiation amorphization in different dose. A series of irradiation experiments of different doses were taken on Mo-8.4Re films. While the ion current density was kept at the same rate of $3 \mathrm{~mA} / \mathrm{cm}^{2}$, the irradiation doses were set as $1 \times 10^{15}$, $2 \times 10^{15}, 3 \times 10^{15}, 4 \times 10^{15}$ and $5 \times 10^{15}$ ions $/ \mathrm{cm}^{2}$.

Figure 2 shows the HRTEM images of Mo-8.4Re alloy film irradiated at different doses: (a) unirradiated; (b) $1 \times$ $10^{15}$ ions $/ \mathrm{cm}^{2}$, (c) $2 \times 10^{15}$ ions $/ \mathrm{cm}^{2}$, and (d) $3 \times 10^{15}$ 


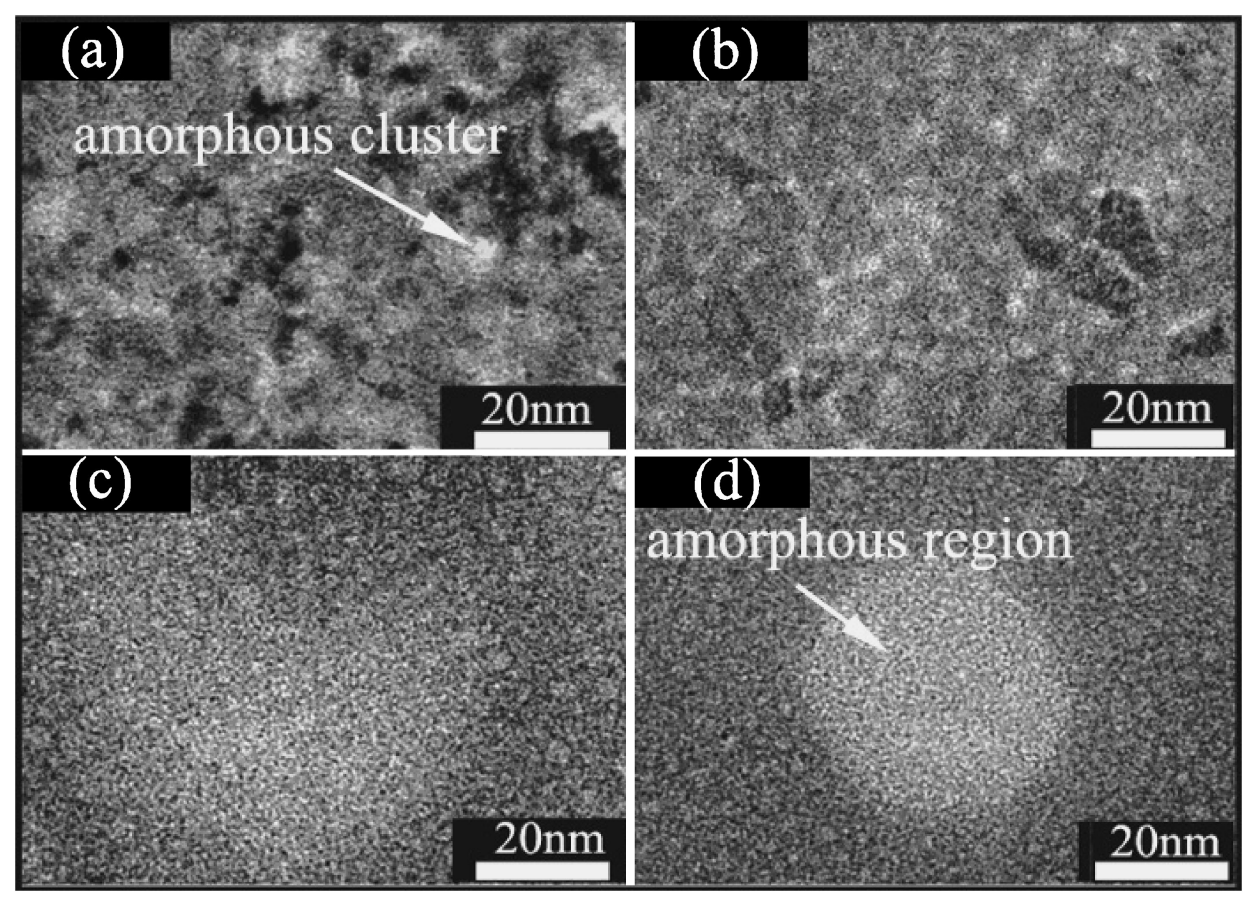

Fig. 3 The forming process of amorphous regions from amorphous clusters while ion dose increasing, HRTEM images of Mo-8.4Re films being irradiated: (a) $2 \times 10^{15}$ ions $/ \mathrm{cm}^{2}$, (b) $3 \times 10^{15}$ ions $/ \mathrm{cm}^{2}$, (c) $4 \times 10^{15} \mathrm{ions} / \mathrm{cm}^{2}$, and (d) $5 \times 10^{15} \mathrm{ions} / \mathrm{cm}^{2}$.

ions $/ \mathrm{cm}^{2}$. The TEM image is taken in a high magnification to observe the irradiation effects in the nano-scale crystal gains. One could see in (a) that the film is made up of grains shown by lattice stripes. The diameter of these grains are $3 \sim 4 \mathrm{~nm}$. However, while irradiated by $\mathrm{Xe}^{+}$, the crystal grains began to collapse. In Fig. 2(b), (c) and (d), it is clear that the areas of lattice stripes got smaller and the grain boundaries of amorphous phase become wider while ion dose increasing. In the irradiation process, the injected ions could induce a series of atomic collisions and keep on knocking the atoms out of the lattice to the disordered area. We could track this process by those HRTEM images. A sketch of the former is also shown in (e), in which it is shown that while more and more atoms were knocked out of the lattice, the amorphous boundary was enlarged and finally grew to an amorphous cluster with the diameter of several $\mathrm{nm}$.

Further transformations of Mo-8.4Re films in higher ion dose were shown in Fig. 3, in which we decreased the magnification in order to investigate the irradiation effects in scale of dozens $\mathrm{nm}$. The ion doses in Fig. 3 are (a) $2 \times$ $10^{15}$ ions $/ \mathrm{cm}^{2}$, (b) $3 \times 10^{15}$ ions $/ \mathrm{cm}^{2}$, (c) $4 \times 10^{15}$ ions $/ \mathrm{cm}^{2}$, and (d) $5 \times 10^{15}$ ions $/ \mathrm{cm}^{2}$. In (a) and (b), we could see that amorphous clusters with diameter of several $\mathrm{nm}$ distributing in the film. While ion dose increased, one could find out in (c) that more amorphous clusters were formed and the intensity of the amorphous clusters was so high that some clusters trended to combine together. Then in (d) the combining trend finally led to the amorphous region with the diameter of $\sim 20 \mathrm{~nm}$. So it could be say that the increase of ion dose firstly induced lattice damage and dislocation of lattice atoms; then the disordered atoms form amorphous clusters in the grain boundaries; and finally the amorphous clusters are combined to amorphous regions.

It is reported that the implantation of ions with high energy could induce irradiation defects and voids both in crystalline and amorphous systems, and finally causes microscopic and even macroscopic swelling. For example, Wang and coworkers reported that the radiation-induced formation of void-like cavities in amorphous germanium, ${ }^{16)}$ Guglya and co-workers found that high dose of helium ion irradiation leads to structure destruction and gas-vacancy void formation in nanocrystalline $\mathrm{Cr}-\mathrm{N}$ coatings, ${ }^{17)}$ and $\mathrm{Hu}$ et al. reported that the irradiation of $\mathrm{Xe}^{+}$could create swelling patterns of nanometer scale on the monocrystalline $\mathrm{Si}$ substrates for the irradiation defects and voids in the irradiated areas. ${ }^{18)}$

In this case, the implantation of $\mathrm{Xe}+$ might also create voids inside the Mo-Re films. In Fig. 3(b), (c), and (d), the small white spots looks like voids of several $\mathrm{nm}$, rather similar with the voids in amorphous germanium. ${ }^{16)}$ However, if we link this to the HRTEM images in Fig. 2, it is obvious that those small white spots grew from the grain boundaries of amorphous phase. So it is inferred that they are amorphous clusters of Mo, Re, and Xe atoms. On the other hand, it is still possible that they are irradiation voids aggregated from defects and Xe atoms. Finally those amorphous clusters or voids become denser and combined at higher ion dose, and induce big amorphous region in Fig. 3(d).

The amorphization process of Mo-8.4Re alloy films were listed in Table 1. As we have already found out the relationship between the irradiation conditions and the irradiation effects, it is possible to forecast the localized amorphization in Mo-Re alloy and avoid the structural failure caused by irradiation induced amorphization.

In summary, irradiation effects of Mo-Re alloy films were investigated by the irradiation of $200 \mathrm{keV} \mathrm{Xe}^{+}$at the dose of $5 \times 10^{15}$ ions $/ \mathrm{cm}^{2}$. The irradiation effects in Mo-8.4Re film such as amorphous regions and grown crystal grains were observed. The energy deposition process and the high irradiation damage, which are both induced by ion irradiation, are considered to be the main causations of the 
Table 1 The amorphization process of Mo-8.4Re alloy films.

\begin{tabular}{|c|c|c|c|c|}
\hline Dose (ions $/ \mathrm{cm}^{2}$ ) & dpa & $\begin{array}{c}\text { Dose rate } \\
\left(\text { ions } / \mathrm{cm}^{2} \cdot \mathrm{s}\right)\end{array}$ & $\begin{array}{l}\text { dpa rate } \\
(\mathrm{dpa} / \mathrm{s})\end{array}$ & Irradiation effect \\
\hline $1 \times 10^{15}$ & 17.83 & $1.88 \times 10^{13}$ & 0.34 & Enlarged grain boundary \\
\hline $2 \times 10^{15}$ & 35.66 & $1.88 \times 10^{13}$ & 0.34 & Amorphous clusters $(2 \sim 4 \mathrm{~nm})$ \\
\hline $3 \times 10^{15}$ & 53.50 & $1.88 \times 10^{13}$ & 0.34 & Amorphous clusters $(2 \sim 4 \mathrm{~nm})$ \\
\hline $4 \times 10^{15}$ & 71.33 & $1.88 \times 10^{13}$ & 0.34 & Combined amorphous clusters \\
\hline $5 \times 10^{15}$ & 89.16 & $1.88 \times 10^{13}$ & 0.34 & Amorphous region $(\sim 20 \mathrm{~nm})$ \\
\hline
\end{tabular}

irradiation effects in Mo-8.4Re alloy films. The detailed amorphization process was also investigated by a series of implantation experiments in different doses. The whole amorphization process including lattice damage, amorphous clusters, and amorphous regions, was related with certain ion doses. Since Mo-Re alloys are attractive candidates for use as structural materials in irradiative applications, this study might be useful for selecting alloy content and controlling irradiation damage.

\section{Acknowledgements}

The authors are grateful to the financial support from the National Natural Science Foundation of China (Grant No. 50701026), and the National Basic Research Program of China (973 program, 2010CB731600).

\section{REFERENCES}

1) S. X. Wang, L. M. Wang, R. Ewing and R. Doremus: J. Appl. Phys. 81 (1997) 587-593.

2) W. Jiang, W. Weber, L. M. Wang and K. Sun: Nucl. Instr. Meth. Phys. Res. B 218 (2004) 427-432.
3) L. M. Wang, S. X. Wang, R. Ewing, A. Meldrum, R. Birtcher, P. Provencio, W. Weber and H. J. Matzke: Mater. Sci. Eng. A 286 (2000) 72-80.

4) B. X. Liu, W. S. Lai and Q. Zhang: Mater. Sci. Eng. R 244 (2000) 1-46.

5) Q. Zhang, Z. C. Li, C. Lin, B. X. Liu and E. Ma: J. Appl. Phys. 87 (2000) 4147-4152.

6) C. McHargue and J. Williams: Nucl. Instr. Meth. Phys. Res. B 80-81 (1993) 889-894.

7) T. Tanabe and S. Muto: Physica Scripta T81 (1999) 104-107.

8) T. Hioki, A. Itoh, M. Ohkubo, S. Noda, H. Doi and J. Kawamoto: J. Mater. Sci. 21 (1986) 1321-1328.

9) M. Iwaki: Particle Beams Plasma Interact. Mater. ion Plasma Surf. Finish. 107 (2004) 107-110.

10) S. Agnew and T. Leonhardt: JOM 55 (2003) 25-29.

11) G. Leichtfried, J. Schneinel and M. Heilmaier: Metall. Mater. Trans. A 37A (2006) 2955-2961.

12) J. Busby, K. Leonard and S. Zinkle: J. Nucl. Mater. 366 (2007) 388406.

13) A. Hasegawa, K. Ueda, M. Satou and K. Abe: J. Nucl. Mater. 258-263 (1998) 902-906.

14) S. Fabritsiev and A. Pokrovsky: J. Nucl. Mater. 252 (1998) 216-227.

15) A. Hasegawa, K. Abe, M. Satou and C. Namba: J. Nucl. Mater. 225 (1995) 259-266.

16) L. M. Wang and R. C. Birtcher: Appl. Phys. Lett. 55 (1989) 2494-2496.

17) A. Guglya, I. Neklyudov and R. Vasikenko: Radi. Effects Defects Solids 162 (2007) 643-649.

18) Y. Hu, Z. C. Li and Z. J. Zhang: Physica E 41 (2009) 833-837. 